\title{
Cost-Effective Extracorporeal Membrane Oxygenation Simulation
}

\author{
Guillaume Alinier $r^{1,2,3}$ \\ ${ }^{1}$ Department of Research, Hamad Medical Corporation \\ Ambulance Service, Doha, Qatar \\ ${ }^{2}$ School of Allied Health Professions, Midwifery and Social Work, \\ University of Hertfordshire, Hatfield, United Kingdom \\ ${ }^{3}$ Faculty of Health and Life Sciences, Northumbria University, \\ Newcastle Upon Tyne, United Kingdom
}

\author{
Address for correspondence Guillaume Alinier, PhD, MPhys, \\ PGCert, SFHEA, CPhys, MIPEM, MInstP, HMC Ambulance Service \\ Headquarter, Medical City, Al Rayyan Road, Doha, Qatar \\ (e-mail: galinier@hamad.qa; g.alinier@herts.ac.uk).
}

Abstract


Keywords
- cost-effective
- extracorporeal mem-
brane oxygenation
- education
- patient safety
- simulation
- training

This invited review article was presented orally on the occasion of the South West Asian Chapter conference of the Extracorporeal Life Support Organization (ELSO) held in New Delhi, India in January 2018. It has an educational focus on the topic of extracorporeal membrane oxygenation (ECMO), which is increasingly being used as a lifesaving bridge therapy. A case is being made regarding the adage "practice makes perfect" to be considered in the context of simulation-based education to ensure patient safety. Technology-enhanced simulation-based deliberate practice should be used more commonly to support clinicians in the development of all their professional skills. This is an ethical imperative that may be addressed using low-cost simulation modalities that are sometimes proven to be as effective as more expensive approaches. Educators can now design their programs according to published best practice standards for the benefit of their learners, and ultimately the patients they care for. Simulation-based education clearly has a place and important role to play in preparing ECMO teams dealing with routine procedures as well as emergency situations. Several solution and approaches are being presented alongside innovative work currently being done in collaboration between a regional ELSO center of excellence and an academic institution. This innovative simulator is composed of several modules serving different functions required for the simulation of ECMO emergencies at a much lower cost than using the real machine and its various expensive disposable components.

\section{Introduction}

Extracorporeal membrane oxygenation (ECMO) is a high-risk but lifesaving therapy that has gained in popularity particularly following the 2009 H1N1 outbreak as it can provide temporary pulmonary and/or cardiac support. ${ }^{1,2}$ A combination of factors determines that ECMO patient mortality rates are significant. ${ }^{3-5}$ These include the fact that patients qualifying for ECMO cannulation are already in an unstable and critical condition, the invasive nature of the procedure involved (generally jugular or femoral venous and/or arterial cannulation), and the potential physiologic or mechanical complications associated with ECMO. An international study showed that hospitals with higher volumes of ECMO patients achieved lower mortality rates than facilities that were dealing with fewer ECMO cases. ${ }^{6}$ The technicality of the procedures involved to initiate and sustain ECMO care is such that it requires an experienced team, and hence the adage of "practice makes perfect" is thought to be a significant contributor to improved patient outcomes. ${ }^{6}$ To that effect, a position paper recommended that to deliver optimal care, a facility should perform at least 20 ECMO cases per year so clinicians can maintain their skills to an appropriate standard.

\section{"Practice Makes Perfect"}

It is logical to think that the more one performs a task, the better one becomes at it, but this is only valid under
DOI https://doi.org/

10.1055/s-0038-1670023.

ISSN 2457-0206.
Copyright @2018 Official Publication of The Simulation Society (TSS), accredited by International Society of Cardiovascular Ultrasound (ISCU).
License terms

() (1) $\odot \circledast$ 
the assumption that the correct technique is used, that one receives feedback to improve their performance, and that the outcome achieved is at least meeting the expected standard. The application of the adage "practice makes perfect" is sometimes assumed under the premise of actually "practicing" the act of providing actual patient care as can be understood from the published recommendation to perform a certain minimum number of ECMO cases per year to ensure optimum patient outcome. ${ }^{1}$ Although still sometimes argued to require more research or evidence, ${ }^{8,9}$ technologyenhanced simulation-based deliberate practice can be used to support skill development and improve performance, and hence could supplement real patient care experiences to provide additional exposure to ECMO clinicians.

The regulatory agencies of most commercially driven high-risk industries, such as aviation and energy production, did not wait to get sufficient evidence to make simulation training a mandatory requirement for most of the concerned professionals and for the facilities involved. ${ }^{10,11}$ Sadly one has to acknowledge that some of the key differences are that if something goes wrong, the life of the professionals involved is at stake, as well as very expensive commercial assets, as opposed to "only" the lives of other individuals. The wider adoption of simulation-based training approaches in the health care sector should become a more widely recognized ethical imperative. Although this ethical aspect has been recognized and published in an article back in 2003, ${ }^{12}$ the simulation developments in health care in terms of adoption at a global scale are still very slow.

In some instances and to alleviate pressures on practice placements, simulation has been accepted to complement or replace a predetermined number of real patient contact hours by some professional bodies. ${ }^{13}$ This is potentially a very favorable opportunity, but it relies on the validity of the simulation-based experiences so participants can transfer their learning in the real clinical setting. Until recently, health care had no proper simulation education standards, but this has now been addressed by relevant learned societies that have also thought of developing certifications for simulation educators and program accreditation requirements to promote high-quality education. ${ }^{14-16}$ These do not necessarily promote expensive over low-cost simulation modalities, but rather put emphasis on the facilitation of relevant and high-quality educational learning opportunities for participants for initial training and continuing professional development.

\section{Focus on Patient Safety}

Since the Institute of Medicine report "To Err Is Human,"17 a real patient safety movement has been initiated. It has also triggered a greater focus on how medical errors are reported and investigated, and how health care professionals are trained. ${ }^{18,19}$ Several strategies can be used to improve an organization's safety culture, but these produce varying effects depending on the context and how they are imple-

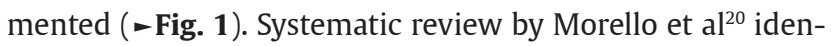
tified a list of targeted patient safety issues, most of which are linked to individual and organizational culture ( - Fig. 2 ).
These can be remembered with the mnemonic "TRRIAL" as elements that can be teased out via careful analysis of situations as a nonpunitive "trial" or an "after action review."21 Improvements in terms of patient safety climate and patient outcomes are not always easy to measure due to many potential confounding factors, but it is what health care institutions need to concentrate on to ensure resources are only allocated to the most effective approaches. Recommendations made to the aviation industry regarding technical and team-based training are directly applicable to health care, notably the notions of Human Factors and Crew or Crisis Resource Management training (CRM). ${ }^{19,22,23}$ Hamad Medical Corporation is making great progress in terms of embedding various simulation modalities within its orientation training programs and courses, bringing up patient safety and CRM-related discussion topics during workshop presentations, team-based assignments, and debriefings with simulation participants. ${ }^{24,25}$ The ultimate objective of these educational interventions is to encourage participants to reflect by considering the issues faced and their approach to tackling the situation.

Some studies have already demonstrated that simulation-based CRM learning was transferable to the clinical setting to improve team performance, safety culture, and patient outcomes. ${ }^{20,26}$ Simulation has also been demonstrated to improve clinicians' performance for a multitude of procedural skills, sometimes with some effect on patient-related outcomes. ${ }^{9,27}$ Evidence of the impact of simulation-based training on ECMO patient care is still very sparse, but it should not preclude its

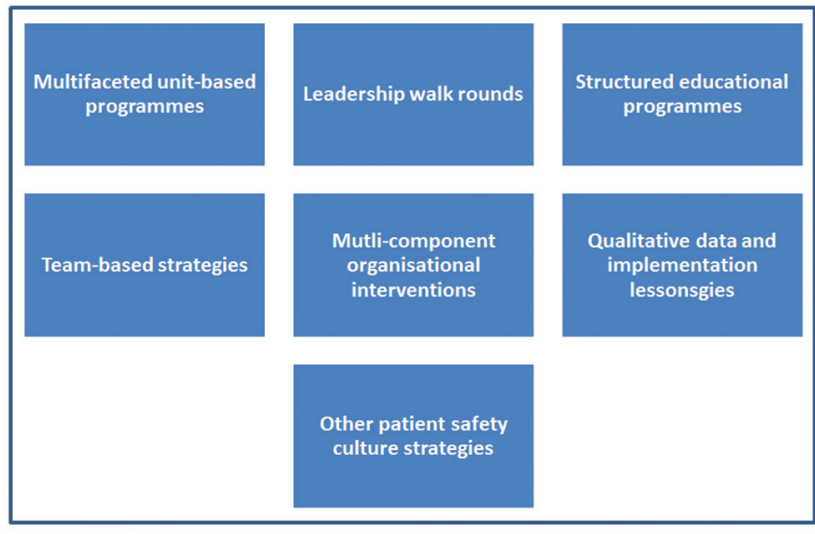

Fig. 1 List of strategies for improving patient safety. ${ }^{20}$

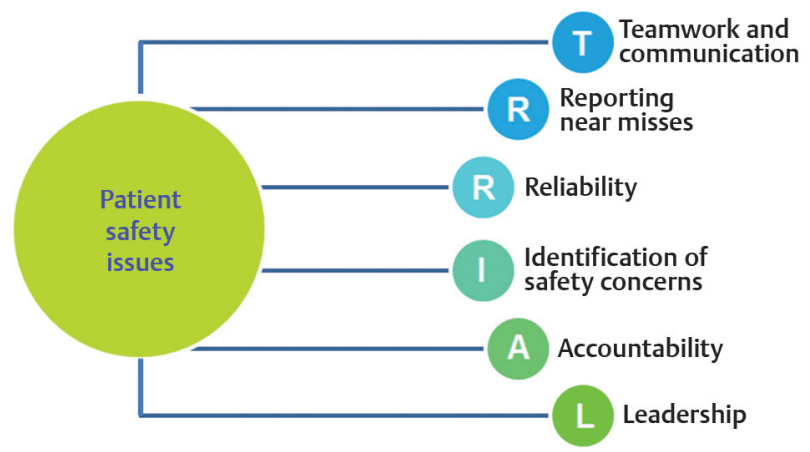

Fig. 2 List of targeted patient safety issues. (Adapted from Morello et al. ${ }^{20}$ ) 
adoption. ${ }^{28-30}$ Well-designed simulation-based ECMO educational programs have the potential to go a long way in promoting safe patient care and developing team members' skills and competencies in dealing with basic ECMO care as well as emergency situations. ${ }^{31-33}$ Simulation clearly has a place and important role to play in preparing ECMO teams dealing with routine procedures as well as emergency situations.

\section{Relevant and Affordable Simulation}

Extracorporeal membrane oxygenation involves very costly equipment and disposable supplies such as the oxygenation membrane, which is a central element of the overall system, and the pump ensuring the circulatory function. The financial aspect of the equipment involved inherently limits the number of such equipment in any given facility and their availability for ECMO training purposes, but it may not necessarily be the case if the expensive components can be substituted by customized replicas that meet the expected functionality without compromising the degree of fidelity or realism where it is required. ${ }^{34}$ Until now, immersive or full-scale ECMO simulation has totally relied on using real medical devices for training purposes, with customized circuits connected to a patient simulator to allow for manipulation of pressures and recreate various patient- or circuit related emergency situations. ${ }^{35-37}$ Some commercial products have also emerged on the market (e.g., Parallel Simulator by Chalice Medical Ltd. and EigenFlow ECMO Simulator by Curtis Life Research), but they also rely on the use of the real ECMO machine and its expensive disposable components. There are also many aspects of ECMO care principles that can be effectively taught and practiced through water drills using the basic circuit components, but it provides a far less immersive and contextualized learning experience than simulation modalities also involving a patient simulator. ${ }^{38,39}$ As soon as a patient simulator is added in the simulation-based learning experience, participants can be exposed to a much greater number of scenarios and a wider spectrum of learning objectives. The scenarios can then be followed by a debriefing period during which the decision-making processes and actions of the participants can be analyzed to develop a better understanding of their mental frames and address the issues identified..$^{40}(-$ Fig. 3 ) illustrates

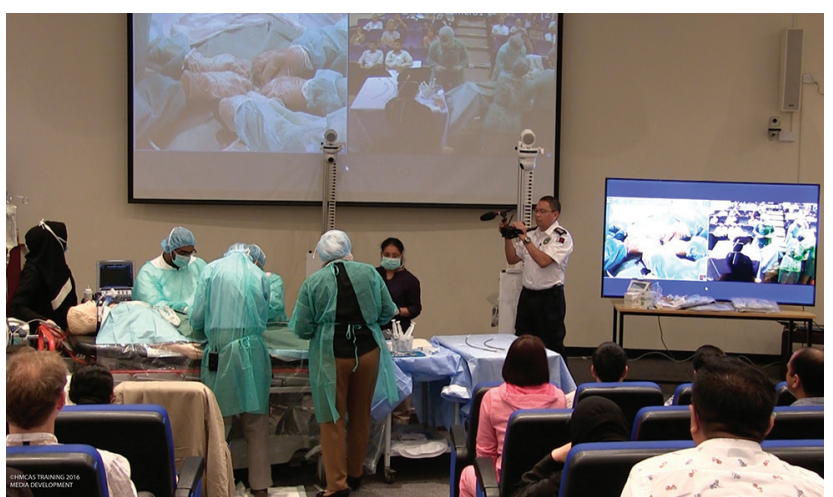

Fig. 3 Demonstration of the full venovenous ECMO cannulation procedure during a respiratory ECMO course held in Doha, Qatar. an ECMO cannulation demonstration in front of a large audience of learners, whereas ( - Fig. 4)shows a multiprofessional team of clinicians taking part in an in situ scenario during which they had to recognize the circuit failure and proceed with the circuit change.

Since 2016, to overcome issues of equipment availability for training purposes and to promote continuous ECMO education opportunities, Hamad Medical Corporation has been collaborating with Qatar University's College of Engineering on the development of innovative ECMO simulation solutions. The team is developing a relatively low-cost yet high-fidelity or high-technology modular ECMO simulator. ${ }^{41}$ The work has been partially supported by some research grants. The ECMO circuit simulator system is based on interconnected modules serving different functions required for simulation of ECMO emergencies and includes simplified three-dimensional (3D) printed replicas of otherwise expensive consumable parts, such as the oxygenation membrane and the actual ECMO machine to enable the circulation of a thermochromic fluid simulating the patient blood. The functional requirements are addressed by mostly nonmedical grade parts such as mechanical pumps, electronic circuitry and sensors, and cloud-based applications that allow to wirelessly create and control realistic scenarios. ${ }^{41}$ Another aspect of this project relates to the development of an ECMO patient simulator, paired with an emulated user controllable patient monitor, that can support all current venous and arterial cannulation configurations in a realistic manner. The simulated blood will also have user controllable venous and arterial pressures and parallel laminar and pulsatile flows to simulate the venous and arterial system. The jugular and femoral sites will be fitted with ultrasound compatible cannulation interchangeable modules of an appropriate texture to enable proper dilatation and venous and arterial cannulation. To allow clinicians to verify the positioning of the ECMO cannulas, the heart anatomy will also be reproduced in an ultrasound friendly material.

We are anticipating that the final solution combining the various modules of the ECMO circuit simulator and the ECMO patient simulator will be a cost-effective platform that can be customized to meet the appearance of any ECMO console and reproduce realistically all the common patient and machine

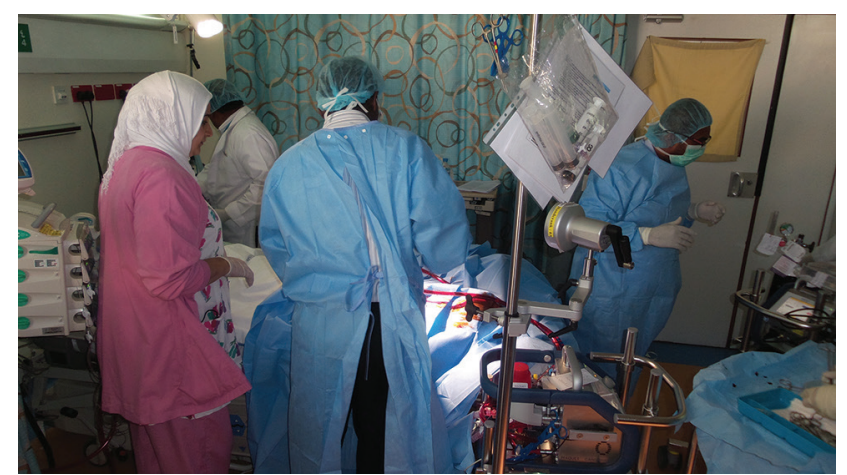

Fig. 4 Full-scale simulation session with a patient simulator adapted for the ECMO procedure (With the kind permission of Sister Arzak Hamed, MICU Charge Nurse/Senior ECMO Nurse Specialist/ECMO Nurse Co-lead, Hamad General Hospital, Doha, Qatar.) 
emergencies. The main elements of the overall proposed ECMO simulator that will generate recurring costs, based on usage, are for the rubber modules used at the cannulation sites and the thermochromic fluid used to simulate the blood. We can estimate that the material cost for the overall solution currently being developed including the patient platform, electronic and mechanical hardware, pumps, thermochromic inks, rubber material, and user interfaces will not exceed U.S. $\$ 2,500$.

The notion of low cost is sometimes associated with low fidelity and probably to be of limited educational benefit or effectiveness, but it is not necessarily the case as it mainly depends on the appropriateness of the fidelity in relation to the learning objectives and on the level of the participants, and how the simulation technology is actually used from an educational point of view..$^{34,42}$ Low cost is a relative and subjective term, when it comes to a complex and expensive medical procedure. Because ECMO is still a relatively rarely used rescue therapy, it remains an expensive procedure, so any savings achieved enabling the facilitation of ECMO simulation-based training using an educationally sound approach and validated tools are advantageous.

\section{Conclusion}

Simulation-based education is still a domain in its infancy at large, and ECMO is a therapeutic procedure that has not yet been globally embraced probably because of logistical or economic barriers. As for all high-risk procedures, especially if they are rarely implemented, optimal competence and skill maintenance of the clinical team should be a high-level priority. Simulation followed by a facilitated debriefing has been identified as an effective approach for clinicians to practice and acquire experience..$^{31,39}$ Although such evidence is limited in the context of ECMO, ${ }^{32}$ it remains nonetheless an important educational strategy that can improve patient safety. There are few commercial options for ECMO simulation and many limitations to these systems and those developed in-house across the globe. ${ }^{37}$ The ECMO simulation system being developed in Qatar is hoped to make a significant impact in this relatively niche market in terms of training opportunities and offerings due to its relative affordability, the modular approach used, and the high level of realism it offers. ${ }^{41}$ Providing a simulator platform that affords rehearsing cases from pre-ECMO patient selection, ECMO circuit troubleshooting, through to decannulation in a highly realistic manner, provides a novel and valuable training opportunity that should significantly contribute to developing the skills of ECMO clinicians and enhance patient safety. Our work is ongoing, and further publications describing our solutions will be published in medical and simulation education journals in the coming year.

\section{Conflict of Interest}

Dr. Alinier has a patent using thermochromic ink for blood simulation in medical training pending to Qatar University, Doha, Qatar.

\section{Acknowledgments}

The author is grateful to the ECMO team from Hamad Medical Corporation, under the leadership of Dr. Ibrahim Fawzy Hassan, and the team of researchers at Qatar University led by Dr. Fayçal Bensaali and Prof. Abbes Amira.

\section{Funding}

This paper was made possible by an UREP grant \#19-0622-026 from the Qatar National Research Fund (a member of Qatar Foundation) and a Grant from Qatar University (QUST-CENG-SPR-15/16-8).

\section{Disclaimer}

The statements made herein are solely the responsibility of the author.

\section{References}

1 Combes A, Brodie D, Bartlett R, et al; International ECMO Network (ECMONet). Position paper for the organization of extracorporeal membrane oxygenation programs for acute respiratory failure in adult patients. Am J Respir Crit Care Med 2014;190(5):488-496

2 Davies A, Jones D, Bailey M, et al; Australia and New Zealand Extracorporeal Membrane Oxygenation (ANZ ECMO) Influenza Investigators. Extracorporeal membrane oxygenation for 2009 influenza A (H1N1) acute respiratory distress syndrome. JAMA 2009;302(17):1888-1895

3 Camboni D, Philipp A, Holzamer A, et al. Ten year institutional results of 465 consecutive adult patients in cardio-pulmonary failure supported with veno-arterial extracorporeal membrane oxygenation. Thorac Cardiovasc Surg 2016;64(1):27

4 Carpenter JL, Yu YR, Cass DL, et al. Use of venovenous ECMO for neonatal and pediatric ECMO: a decade of experience at a tertiary children's hospital.Pediatr Surg Int 2018;34(3):263-268

5 Posluszny J, Rycus PT, Bartlett RH, et al; ELSO Member Centers. Outcome of adult respiratory failure patients receiving prolonged ( $\geq 14$ days) ECMO. Ann Surg 2016;263(3):573-581

6 Barbaro RP, Odetola FO, Kidwell KM, et al. Association of hospital-level volume of extracorporeal membrane oxygenation cases and mortality. Analysis of the extracorporeal life support organization registry. Am J Respir Crit Care Med 2015;191(8):894-901

7 Ericsson KA. Deliberate practice and the acquisition and maintenance of expert performance in medicine and related domains. Acad Med 2004;79(10, Suppl):S70-S81

8 Marcus H, Vakharia V, Kirkman MA, Murphy M, Nandi D. Practice makes perfect? The role of simulation-based deliberate practice and script-based mental rehearsal in the acquisition and maintenance of operative neurosurgical skills. Neurosurgery 2013;72(1, Suppl 1):124-130

9 Sturm LP, Windsor JA, Cosman PH, Cregan P, Hewett PJ, Maddern GJ. A systematic review of skills transfer after surgical simulation training. Ann Surg 2008;248(2):166-179

10 Page RL. Brief history of flight simulation. Proceedings 2000:11-17

11 Golding D, Kasperson RE. Emergency planning and nuclear power: looking to the next accident. Land Use Policy 1988;5(1):19-36

12 Ziv A, Wolpe PR, Small SD, Glick S. Simulation-based medical education:anethical imperative. AcadMed 2003;78(8):783-788

13 Nursing and Midwifery Council. Supporting Direct Care through Simulated Practice in the Pre-registration Programme. NMC Circ 2007 
14 Lioce L, Meakim CH, Fey MK, Chmil JV, Mariani B, Alinier G. Standards of best practice: simulation standard IX: simulation design. Clin Simul Nurs 2015;11(6):309-315

15 Sittner BJ, Aebersold ML, Paige JB, et al. INACSL standards of best practice for simulation: past, present, and future. Nurs Educ Perspect 2015;36(5):294-298

16 Alinier G, Platt A. International overview of high-level simulation education initiatives in relation to critical care. Nurs Crit Care 2014;19(1):42-49

17 Kohn LT, Corrigan JM, Donalson MS. To Err Is Human: Building a Safer Health System. Washington DC: National Academy Press; 1999

18 Kaldjian LC, Jones EW, Wu BJ, Forman-Hoffman VL, Levi BH, Rosenthal GE. Reporting medical errors to improve patient safety: a survey of physicians in teaching hospitals. Arch Intern Med 2008;168(1):40-46

19 Helmreich RL. On error management: lessons from aviation. BMJ 2000;320(7237):781-785

20 Morello RT, Lowthian JA, Barker AL, McGinnes R, Dunt D, Brand C. Strategies for improving patient safety culture in hospitals: a systematic review. BMJ Qual Saf 2013;22(1):11-18

21 Sawyer TL, Deering S. Adaptation of the US Army's After-Action Review for simulation debriefing in healthcare. Simul Healthc 2013;8(6):388-397

22 Gaba D, Howard SK, Fish KJ, Smith BE, Sowb Y. Simulation-based training in Anesthesia Crisis Resource Management (ACRM): a decade of experience. Simul Gaming 2001;32(2):175-193

23 Logarajah S, Alinier G. An integrated ABCDE approach to managing medical emergencies using CRM principles. J Paramed Pract 2014;6(12):620-625

24 Gangaram P, Alinier G, Menacho A. Crisis resource management in emergency medical settings in Qatar. Int Paramed Pract 2017;7(2):18-23

25 Alinier G, Hamed A, Racela B. ECMO transport simulation. Qatar Med J 2017;1:60

26 Boet S, Bould MD, Fung L, et al. Transfer of learning and patient outcome in simulated crisis resource management: a systematic review. Can J Anaesth 2014;61(6):571-582

27 Cook DA, Hatala R, Brydges R, et al. Technology-enhanced simulation for health professions education: a systematic review and meta-analysis. JAMA 2011;306(9):978-988

28 Brum R, Rajani R, Gelandt E, et al. Simulation training for extracorporeal membrane oxygenation. Ann Card Anaesth 2015;18(2):185-190

29 Brazzi L, Lissoni A, Panigada M, et al. Simulation-based training of extracorporeal membrane oxygenation during H1N1 influenza pandemic: the Italian experience. Simul Healthc 2012;7(1):32-34
30 Zakhary BM, Kam LM, Kaufman BS, Felner KJ. The utility of high-fidelity simulation for training critical care fellows in the management of extracorporeal membrane oxygenation emergencies: a randomized controlled trial. Crit Care Med 2017;45(8):1367-1373

31 Labib A, Alinier G. Can simulation improve ECMO care? Qatar Med J 2017. DOI: 10.5339/qmj.2017.swacelso.7 [epub ahead of print]

32 Burkhart HM, Riley JB, Lynch JJ, et al. Simulation-based postcardiotomy extracorporeal membrane oxygenation crisis training for thoracic surgery residents. Ann Thorac Surg 2013;95(3):901-906

33 Anderson JM, Murphy AA, Boyle KB, Yaeger KA, Halamek LP. Simulating extracorporeal membrane oxygenation emergencies to improve human performance. Part II: assessment of technical and behavioral skills. Simul Healthc 2006;1(4):228-232

34 Tun JK, Alinier G, Tang J, Kneebone RL. Redefining simulation fidelity for healthcare education. Simul Gaming 2015;46(2):159-174

35 Anderson JM, Boyle KB, Murphy AA, Yaeger KA, LeFlore J, Halamek LP. Simulating extracorporeal membrane oxygenation emergencies to improve human performance. Part I: methodologic and technologic innovations. Simul Healthc 2006;1(4):220-227

36 Puślecki M, Ligowski M, Kiel M, et al. ECMO therapy simulator for extracorporeal life support. Am J Emerg Med 2018;36(3):506-508

37 Alinier G, Hassan IF, Alsalemi A, et al. Addressing the challenges of ECMO simulation. Perfusion 2018;33(7):568-576

38 Allison PL, Kurusz M, Graves DF, Zwischenberger JB. Devices and monitoring during neonatal ECMO: survey results. Perfusion 1990;5(3):193-201

39 Montero S, Combes A, Schmidt M. The extracorporeal membrane oxygenation (ECMO) high-fidelity simulator: the best complementary tool to learn the technique. J Thorac Dis 2017;9(11):4273-4276

40 Oriot D, Alinier G. Pocket Book for Simulation Debriefing in Healthcare Cham, Switzerland: Springer International Publishing AG; 2018

41 Al Disi M, Alsalemi A, Alhomsi Y, Bensaali F, Amira A, Alinier G. Revolutionizing ECMO simulation with affordable yet highFidelity technology. Am J Emerg Med 2017;36(7):1310-1312

42 Munshi F, Lababidi H, Alyousef S. Low- versus high-fidelity simulations in teaching and assessing clinical skills. J Taibah Univ Med Sci 2015;10(1):12-15 